\title{
Simulation Experiment Implementation for Coordinative effectiveness Evaluation Method of Air Defense Missile Equipment System
}

\author{
Zheng Wang \\ College of Information System and Management, National University of Defense Technology \\ Changsha, Hunan, China
}

Keywords: Simulation Experiment, Effectiveness Evaluation.

\begin{abstract}
Research on operation effectiveness evaluation method of equipment system is an important part of equipment system demonstration. Due to the complexity of equipment system effectiveness evaluation, a new challenge is put forward to conventional combat simulation. Based on the operation effectiveness evaluation method of equipment system in combination with data farming and data mining, XSIM general simulation modeling and application platform is used to implement simulation experiment for a coordinative effectiveness evaluation method of air defense missile equipment system.
\end{abstract}

\section{Introduction}

With the development of weapon equipment technology, the battlefield environment is more and more complex, and the countermine characteristic of weapon equipment system is increasingly obvious. Operation effectiveness of system is not determined by individual equipment performance and simple summation of the quantity of various equipments within system, but it is the result that various equipments cooperate, coordinate and interact with each other. Only if advanced weapon equipment forms the scientific and reasonable system, it can maximize the overall operation effectiveness in war. The system operation effectiveness analysis is that through analyzing effects of all factors (the quantity of equipment, performance, etc.) on operation effectiveness, the interrelation between key factors is determined to provide a basis for scientific planning and rational construction of weapon equipment system.

Currently, there are mainly two operation effectiveness modeling methods of air defense missile equipment system, i.e. analysis method and simulation method. The selection of method relies on analysis purpose, accuracy requirement and pre-existing conditions. Analysis method is characterized by the computation of effectiveness indexes based on the analytical formula of function relationship between effectiveness and given conditions (generally, performance indexes of low-level system). This analytical formula can be established directly based on Military Operation Research theory and be obtained by using mathematical method to solve the established equation. The common methods contain index method, analytic hierarchy process, fuzzy mathematical method, ADC, etc. Features of analysis method are that good transparency of formula, easy to understand, simple computation, capable of analyzing the relationship between variables, a good mastery of key factors and the change rule between it and operation effectiveness, and convenient for application. Therefore, as long as reliable mathematical model can be established and data input is complete, analysis method is a good modeling method. Analysis method is widely applied in combat modeling [1-3]. For example, the queueing theory method is used to establish the firing effectiveness model of air defense missile firing the target [1]; Markov chain decision analysis method is used to establish the effectiveness model against the situation that tactical missile fires a single target [2]; ADC is used to establish the effectiveness evaluation model for air defense missile weapon equipment system, and uses the expert evaluation method and the grey evaluation method to estimate parameters in the model, in order to complete the effectiveness evaluation of air defense missile weapon equipment system[3]. The disadvantage of analysis method is that the established model is often fairly abstract, poorly vivid, and effective only under limited assumptions. 
Simulation method is that computer simulation is used as experiment means, combat simulation experiment is made by operating model under given conditions, and estimated values of effectiveness indexes are provided after data about combat processes and results from experiments can be processed directly or statistically. With the development of simulation technology, simulation method is also widely applied in air defense operation modeling [4] [5]. From the overall situation of air defense operation, operation elements are simplified, combat units are divided into three types of combat agents, i.e. attacking aircraft, command and control system and surface-to-air missile; Blue is defender, represented by command and control system and surface-to-air missile, while red is attacker, represented by attacking aircraft; Effects of command and control system on air defense operation effectiveness are evaluated by simulation and analysis [6]. Simulation method can detailedly consider all kinds of factors that influence the operational process, so it is suitable for estimation and evaluation of effectiveness indexes for weapon equipment system or operation scheme. However, the modeling difficulty of simulation method is great, the test period is long, and the running time of simulation is very long when factors involved in the model are relatively much more.

\section{Coordinative Effectiveness Evaluation Method of Equipment System}

Sorting evaluation method based on ideal point method and grey correlation is suitable for weapon equipment system. When specific operational mission id performed, there are different operational schemes for selection, which needs to sort schemes to select the best [7]. In two-dimensional space, effectiveness indexes and their values can constitute different curves. This sorting evaluation method comprehensively considers the distance between curves and the shape of curves to sort all schemes. At first, indexes are normalized. Generally, weapon equipment system contains a great number of effectiveness indexes. Effectiveness indexes have different dimensions. Sometimes, the difference between orders of magnitude is large. If original effectiveness indexes are used directly to make evaluation, final evaluation results tend to be effectiveness indexes whose order of magnitude is large. Meanwhile, in order to compare each evaluation results, evaluation results are limited in [0,1], and values of indexes need to be normalized. Then, the weight of effectiveness indexes is determined, and normalized matrix is weighted. Next, reference schemes are determined. There are many types for effectiveness indexes, i.e. efficiency index, cost index, fit index, etc. Regardless of which type of effectiveness indexes, all have positive and negative ideal values. In ideal schemes, for efficiency index, the bigger, the better; for cost index, the smaller, the better; for fit index, the closer to the fit value, the better. Correspondingly, in negative ideal schemes, for efficiency index, the smaller, the better; for cost index, the bigger, the better; for fit index, the farther from the fit value, the better. Then, the difference between evaluation schemes and positive and negative ideal schemes is calculated. The absolute difference matrix, max difference and min difference between evaluation schemes and ideal schemes are calculated. Grey correlation degree between the ith scheme and positive and negative ideal schemes is calculated. Nondimensionalized distance and grey correlation degree are considered comprehensively. Relative similarity degree of scheme is calculated. They are sorted according to similarity degree. The larger the relative similarity degree is, the better the evaluation scheme is; otherwise, the worse.

In order to make weapon equipment maximize combat capability in system countermine, many schemes of system need to be sorted comprehensively. Whether the sorting method is used reasonably is directly related to whether evaluation result is credible. In the sorting evaluation of many schemes of system, the more considered factors are, the more comprehensive evaluation results are. Based on ideal point method and sorting evaluation method of grey correlation, the position relationship between curves and the shape of curves are used as the basis of evaluation, instead of the distance between curves and the change situation being simply used as the basis of scheme evaluation. Its physical meaning is definite, and its evaluation result is more comprehensive and reasonable. 


\section{Experiment Design}

(1) Case Design

Under specific background of air defense anti-missile mission, experiment cases are designed to verify the evaluation method of coordinative effectiveness for equipment system. A force relies on the superior mobile, invisible and attacking performances of the fifth generation of F22 fighter, attempting to violate our airspace for investigation activities and despising our combat capability. Our army has learned the intelligence of action line of the hostile forces, in order to combat the arrogance of the hostile forces and enhance our defense capability. Our army decides to fight back, authorizes an air defense command center in a theater to deploy networked air defense anti-missile system in the enemy investigation route area, and is determined to minimize time, manpower, weapons and other resources to destroy the aircraft. See Table 1 for forces deployment conditions of red and blue party.

Table 1 Forces Deployment of Red and Blue Party

\begin{tabular}{|c|c|c|}
\hline $\begin{array}{c}\text { Forces } \\
\text { deployment }\end{array}$ & $\begin{array}{c}\text { The blue party is an airport, assigned a flight formation of four F22 fighters (each } \\
\text { carries 1 airborne radar, 1 missile warning device and } 2 \text { anti-radiation missiles); the } \\
\text { red party is composed of 1 C300 air defense position unit, 2 early warning radar } \\
\text { positions, 1 theater air defense command center and 1 stand-by AWACS (carrying 1 } \\
\text { airborne radar). Air defense position unit is fitted with 1 radar and 8 surface-to-air } \\
\text { missiles. }\end{array}$ \\
\hline $\begin{array}{c}\text { Deployment } \\
\text { methods }\end{array}$ & $\begin{array}{c}\text { Assume the early-warning radar position of the red party is arranged with good } \\
\text { cooperation, i.e., having early-warning detection capabilities over the incoming target } \\
\text { lines; air alert AWACS conducts early warning detection in the air defense region. If } \\
\text { the blue party is detected, the early warning radar and AWACS will provide guidance } \\
\text { for the air defense position to conduct target tracking and attacking; the fighter of the } \\
\text { blue party flies over the hybrid group of air defense positions according to the default } \\
\text { route and through the early warning radar detection range intersection of the red party } \\
\text { to start airborne radar to search the red party. }\end{array}$ \\
$\begin{array}{c}\text { When the red party destroys the aircraft of the blue party, the interception is } \\
\text { successful; if the blue party breaks the defense of the red party or destroys the } \\
\text { command post, the blue party wins. }\end{array}$ \\
rules
\end{tabular}

Experimental cases include joint optimal guidance and coordinative operation cases of fighter formation in the networked air defense anti-missile system operation. Each case includes detailed experiment design, simulation configuration. Chapter five shows specific analysis of simulation results and two-dimensional situation representation, etc. In the networked air defense anti-missile system operation, one aircraft of F22 flight formation is designed to perform penetration mission.

(a) Networked air defense anti-missile

Optimal anti-missile: When the aircraft enters into the weapon range, if two radars are tracking at the same time, which is chosen by the system to undertake guidance tasks. The experiment configuration of optimal guidance is shown in Table 2.

Table 2 Networked air defense anti-missile experiment settings

\begin{tabular}{|c|c|c|c|c|}
\hline $\begin{array}{l}\text { Experiment } \\
\text { object }\end{array}$ & \multicolumn{4}{|c|}{ Networked air defense anti-missile } \\
\hline $\begin{array}{l}\text { Experiment } \\
\text { method }\end{array}$ & \multicolumn{4}{|c|}{$\begin{array}{l}\text { The radar parameters of the two radar nodes in different places are as follows. Assume the } \\
\text { various indexes of No. } 1 \text { radar are better than those of No. } 2 \text { radar, which is chosen by the } \\
\text { system to undertake guidance tasks. }\end{array}$} \\
\hline \multirow{3}{*}{$\begin{array}{l}\text { Experiment } \\
\text { conditions }\end{array}$} & \multirow{3}{*}{$\begin{array}{l}\text { Parameter settings of early warning } \\
\text { radar (red party) }\end{array}$} & $\begin{array}{l}\text { Properties } \\
\text { Model }\end{array}$ & $\begin{array}{c}\text { Range } \\
\text { resolution }\end{array}$ & $\begin{array}{l}\text { Angular } \\
\text { resolution }\end{array}$ \\
\hline & & $\mathrm{ID}=$ No. 1 radar & 10 & 30 \\
\hline & & $\mathrm{ID}=$ No. 2 radar & 10 & 25 \\
\hline
\end{tabular}

(b) Penetration of fighter formation 
Formation penetration: If the flight formation must fly over an air defense position, how to implement low altitude penetration by formation coordination. See table 2 for formation penetration experiment configuration.

Table 3 Penetration experiment settings of fighter formation

\begin{tabular}{|c|c|}
\hline $\begin{array}{c}\text { Experiment } \\
\text { object }\end{array}$ & \multicolumn{1}{c|}{ Penetration of fighter formation } \\
\hline $\begin{array}{c}\text { Experiment } \\
\text { method }\end{array}$ & $\begin{array}{c}\text { The blue party assigns a flight formation of four F22 fighters to breach the defense } \\
\text { system composed of an air defense position unit and an AWACS, in order to } \\
\text { observe the damage situations of its penetration. Two fighters attack AWACS and } \\
\text { air defense position respectively at first, and the rest of two fighters fly over defense } \\
\text { position of red party after assault is successful. }\end{array}$ \\
\hline $\begin{array}{c}\text { Experiment } \\
\text { conditions }\end{array}$ & $\begin{array}{c}\text { If it is destroyed utterly or its ammunition is exhausted, the penetration of blue party } \\
\text { is failed and it exits the simulation; If there is at least a F22 fighter in the formation } \\
\text { of blue party flying over the position of red party, the defense of red party is failed } \\
\text { and it exits the simulation. }\end{array}$ \\
\hline
\end{tabular}

(2) Simulation Platform and Secondary Development Framework Design

XSIM is a general simulation modeling and application platform; it is a mature simulation application platform and a professional simulation development platform. As an application platform, it has a series of tools and professional model libraries, and supports user to utilize the existing resources to directly carry out research, demonstration, experiment and training work; As a development platform, XSIM provides sophisticated modeling framework and application framework, and supports user to develop model and application system. XSIM platform framework utilizes multilayer design and separates "problem domain" from "simulation domain" completely to make its framework more flexible and extensible.

Its engine is based on the component modeling and discrete event simulation technology. It uses hybrid propulsion based on time and discrete event, utilizes the multithreading concurrency and event synchronization mechanism technology, and can make full use of muti-CPU and multi-core resources of computer. Therefore, it is a centralized and step-by-step general simulation engine. Simulation engine mainly consists of event manager, time manager and other platform services, as shown in Fig. 1. Simulation engine is responsible for the creation and maintenance of other managers and services, including: identifier manager, battlefield manager, service manager, object manager, simulation monitor and object monitoring service, data acquisition service, damage judgement service, commissioning service, transmission service, distributed center service, distributed relay service, etc.

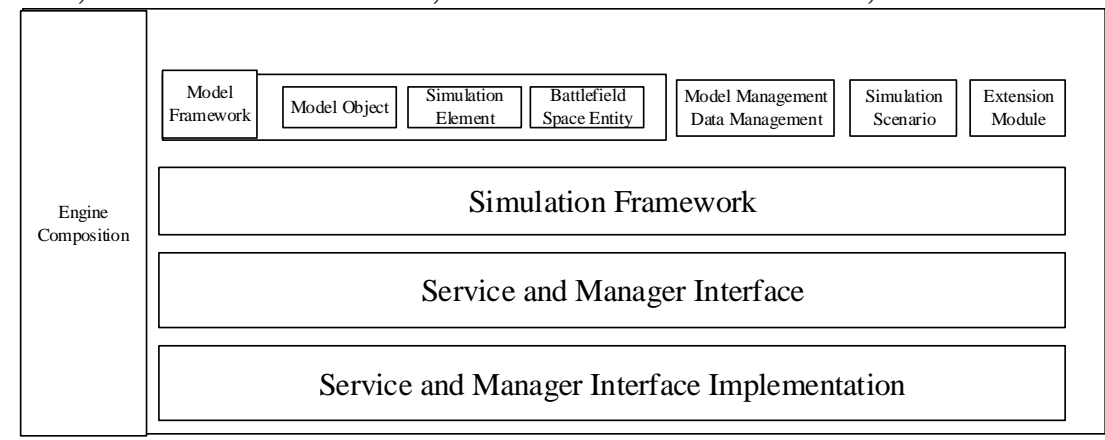

Fig. 1 XSIM engine module composition

Model framework is used to solve problems of model simulation domain, mainly including model object, simulation element, and battlefield space entity. They provide interaction and setting of model and basic data, or get simulation framework to submit and process simulation event and conduct model initialization, etc. Model management and data management are responsible for the load of published model in model library and provide basic data support for the load of scenario. Simulation scenario is used to record simulation intervention entity, resources and other elements, and describe the relationship between entities, simulation duration, step size and other information. Simulation framework is used to solve the problem of uncertainty in manager or service needs when the engine 
drives different scenarios. In XSIM, Simulation framework manages service or manager based on interface, while specific realized key function contains time management, event management, data acquisition, etc. This experiment is conducted on the basis of XSIM general simulation modeling platform.

\section{Simulation Sample Generation based on Data Farming}

The simulation model of a fighter fleet based on Analytic Hierarchy Process (AHP) is used against fighter formation. After normalized, above data farming is used to obtain data, as shown in table 4. Data farming process is a closed loop with analysis and verification, thus the reliability of obtained sample data based on data farming is guaranteed.

Table 4: Farming and harvesting the performance index of fighter formation

\begin{tabular}{|c|c|c|c|c|c|c|c|}
\hline Normalized Index & 1 & 2 & 3 & 4 & 5 & 6 & 7 \\
\hline Situation cognition quality & 0.843 & 0.848 & 0.862 & 0.873 & 0.892 & 0.910 & 0.917 \\
\hline Decision-making time & 0.672 & 0.663 & 0.691 & 0.682 & 0.695 & 0.624 & 0.683 \\
\hline Information acquisition capability & 0.878 & 0.862 & 0.891 & 0.885 & 0.902 & 0.856 & 0.883 \\
\hline Information distribution capability & 0.786 & 0.842 & 0.792 & 0.845 & 0.746 & 0.867 & 0.891 \\
\hline Information protection capability & 0.787 & 0.702 & 0.702 & 0.690 & 0.791 & 0.675 & 0.658 \\
\hline Coordination reliability & 0.823 & 0.832 & 0.834 & 0.823 & 0.845 & 0.817 & 0.825 \\
\hline Coordination credibility & 0.889 & 0.901 & 0.875 & 0.886 & 0.903 & 0.872 & 0.863 \\
\hline Target detection probability & 0.740 & 0.772 & 0.709 & 0.700 & 0.724 & 0.673 & 0.712 \\
\hline Target acquisition probability & 0.532 & 0.459 & 0.527 & 0.541 & 0.519 & 0.473 & 0.509 \\
\hline Probability of kill & 0.583 & 0.571 & 0.585 & 0.498 & 0.519 & 0.536 & 0.572 \\
\hline
\end{tabular}

Mass data are obtained base on above data farming technology. On this basis, typical algorithm, Apriori, can be used to obtain the association relationship of related data. Specific association relationship is shown in Table 5. As seen in Table 5, decision-making time is associated with information acquisition capability, information protection capability is associated with coordination reliability, etc.

Table 5: Association relationship between different indices

\begin{tabular}{|c|c|c|c|c|}
\hline & $\begin{array}{c}\text { Situation } \\
\text { cognition quality }\end{array}$ & $\begin{array}{c}\text { Decision-making } \\
\text { time }\end{array}$ & $\begin{array}{c}\text { Coordination } \\
\text { reliability }\end{array}$ & $\begin{array}{c}\text { Coordination } \\
\text { credibility }\end{array}$ \\
\hline $\begin{array}{c}\text { Information acquisition } \\
\text { capability }\end{array}$ & associated & & \\
\hline $\begin{array}{c}\text { Information } \\
\text { distribution capability }\end{array}$ & & associated & associated \\
\hline $\begin{array}{c}\text { Information protection } \\
\text { capability }\end{array}$ & associated & associated & associated & associated \\
\hline $\begin{array}{c}\text { Target detection } \\
\text { probability }\end{array}$ & & & & \\
\hline
\end{tabular}

\section{Simulation Experiment Results}

(1) Networked Air Defense Anti-Missile

Fig. 2 shows the forces deployment of the red party and the blue party in optimal guidance of case 1 . As shown in Fig. 2, the red party deploys one air defense command center, two ground-based early warning radars and one air defense position unit. A F22 of the blue party is breaking out into the red party. 


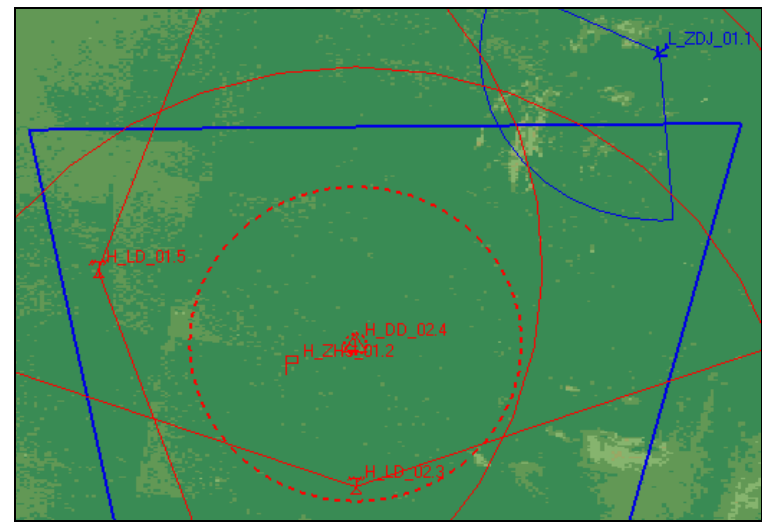

Fig. 2 Basic Deployments of the Red Party and the Blue Party in Optimal Guidance

As shown in Fig. 3 (a), the aircraft of the blue party has successively entered into the detection ranges of two ground-based early warning radars of the red party. The range resolution of No. 2 radar is more than that of No. 1 radar and the aircraft of the blue party firstly enters into the detection range of No. 2 radar, so No. 2 radar firstly starts up and detects targets.

No. 2 radar then transmits preliminary information of threat object to the regional command and control center through the communication network, and is informed that the threat target is approaching. The air defense command center preferentially chooses No. 2 radar to carry out target tracking, at meantime informs the ground air defense unit of the incoming situations according to the indication of incoming targets and releases fire control radar starting command. The fire control radar of ground air defense unit starts up and conducts detection and tracking in guiding airspace.

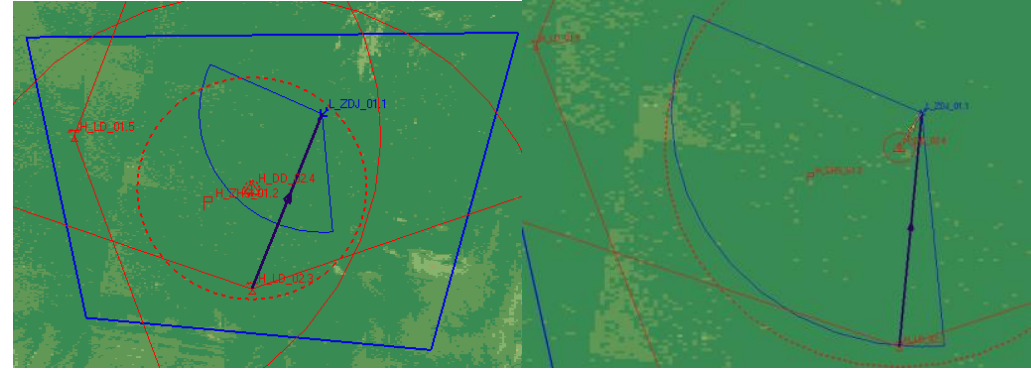

(a)

(b)

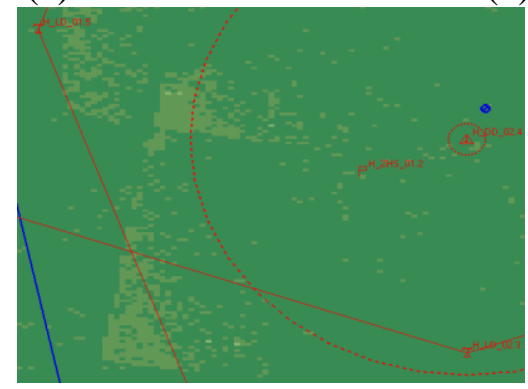

(c)

Fig. 3 Basic Operational Process of Optimal Guidance

After being hit, the combat unit should immediately enter into the launch preparation phase to rapidly conduct fire distribution and transmit the command to the missile launch system. As shown in Fig. 3 (b), the F22 fighter of the blue party enters into the fire range of ground air defense unit of the red party under the target early warning of the No. 2 ground-based early warning radar. As the combat unit of the red party has only one air defense position, it is hit. According to the command of the air defense command and control center, the air defense unit rapidly conducts fire distribution, binds the launching data and launch missile after receiving the fire command of command and control center. The launched missile selects the best fire-control radar for guidance and destroys the invasive fighter of the blue party under the target guidance of the fire control radar and early warning radar of the air defense unit. The blue party does not reach the operational purpose to destroy the command post of the red party and implement fire penetration, as shown in Fig. 3 (c). 
(2) Penetration of Fighter Formation

Fig. 4 shows the forces deployment of the red party and the blue party in optimal guidance of case 2. As shown in Fig. 4, red party deploys a land-based air defense missile position and a stand-by AWACS hovering near missile position. The blue party assigns a flight formation of four F22 fighters to break through air defense missile position of red party. Since the detection distance of radar in air defense missile position is insufficient, AWACS generally assists it to monitor vital area.

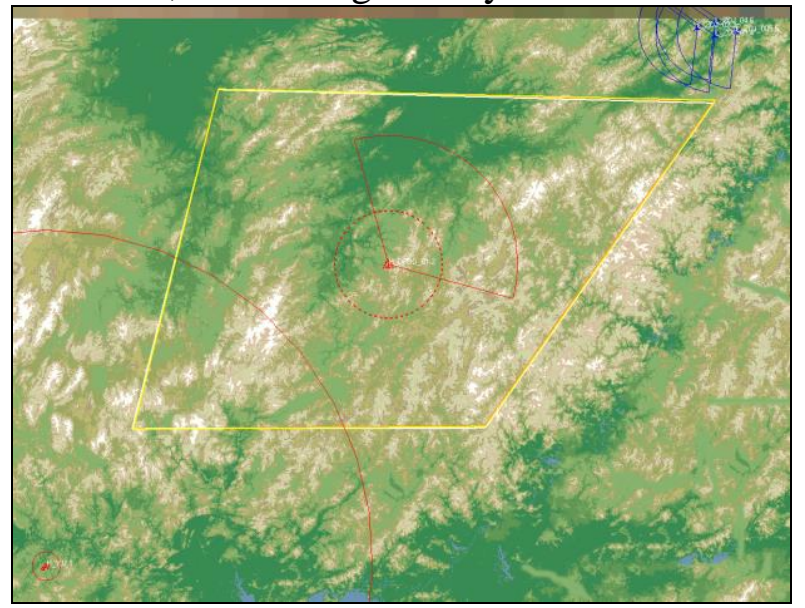

Fig. 4 Formation penetration deployment of red party and blue party

As shown in Fig. 5 (a), since the formation of blue party uses the stealth capability of fighter and the low-altitude penetration tactics, it can avoid the early detection of AWACS, causing the start time of radar in surface-to-air missile position to be later. No.3 fighter of blue party is earlier than missile position to execute anti-radiation attack. As shown in Fig. 5 (a), target is not hit in the first attack, fighter of blue party is locked in air defense position, air defense missile is launched and two are destroyed successfully. In the formation penetration mission of blue party, the goal is achieved, and the central region of red party continues to be entered. In the escape process, AWACS of red party is shot down by No.4 fighter of blue party, because its maneuvering speed is lower than that of blue party, as shown in Fig. 5 (c) and (d).

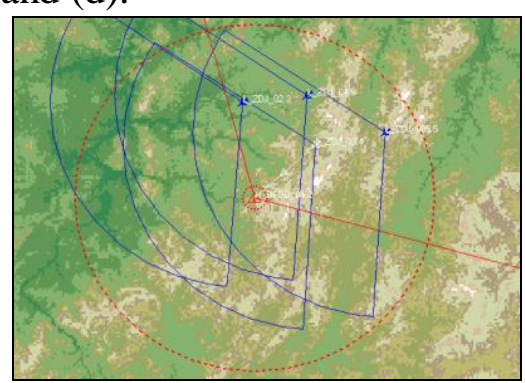

(a)

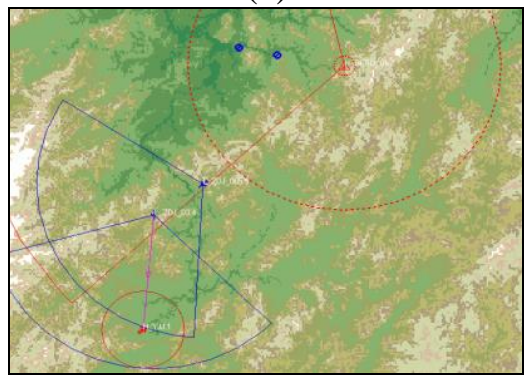

(c)

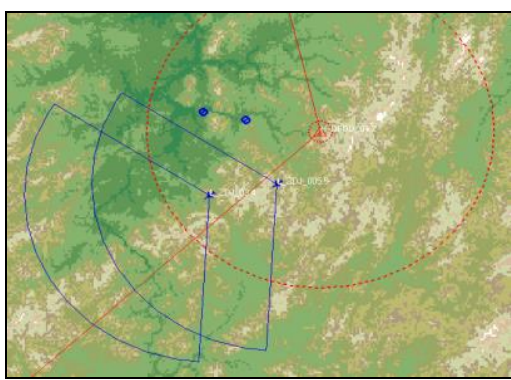

(b)

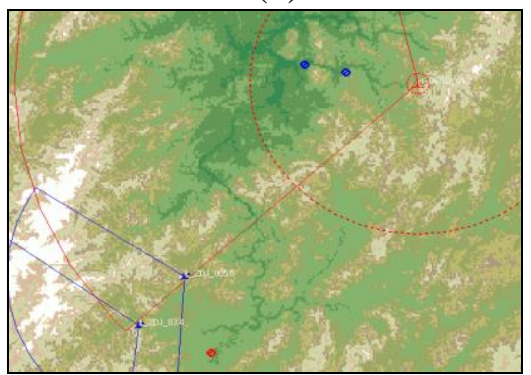

(d)

Fig. 5 Basic operation process of formation penetration

\section{References}


[1] Qing Yang. The Analysis of Queue Property of the Shooting Effectiveness of Air Defense System. Nanjing University of Science and Technology, 2006.

[2] Dewu Wang, Minle Wang, etc. The Markov Chain Decision Analysis Model of the One-target Shooting Effectiveness of Tactical Missiles. The Seminar of Multi-Objective Decision Making, 2005:166-170.

[3] Wenqi Chen. The Evaluative Analysis of the Combat Effectiveness of Air Defense Weapon Systems. University of Xiamen, 2006.

[4] Decheng Zhu, Xueshan Luo, Xueshi Shen. The Application of CPN in Sea-based Air Defense Modeling. Fire Control and Command Control, 2002, 27(3):42-45.

[5] Xiaoyi Wang, Zaiwen Liu, etc. The Multi-objective Optimization of the Allocation Modeling and Decision Making of Air Defense Weapons. Journal of China Ordnance, 2007, 28(2):228-231.

[6] Qiang Liu, Huifeng Xue. Research of Air Defense Effectiveness Evaluation Simulation of based on Multi-agent Command and Control System. Micro-electronics and Computer, 2008, 25(2):126-128.

[7] Qingyue Ma. Research on Integrated Evaluation Techniques of Weapon Equipment System. Harbin Institute of Technology, 2015. 